\title{
Automatically Adapting Home Lighting to Assist Visually Impaired Children
}

\author{
Euan Freeman, Graham Wilson and Stephen Brewster \\ Multimodal Interaction Group \\ Glasgow Interactive Systems Group \\ University of Glasgow, Scotland \\ euan.freeman@glasgow.ac.uk \\ graham.wilson@glasgow.ac.uk \\ stephen.brewster@glasgow.ac.uk \\ http://mig.dcs.gla.ac.uk
}

\begin{abstract}
For visually impaired children, activities like finding everyday items, locating favourite toys and moving around the home can be challenging. Assisting them during these activities is important because it promotes independence and encourages them to use and develop their remaining visual function. We describe our work towards a system that adapts the lighting conditions at home to help visually impaired children with everyday tasks. We discuss scenarios that show how they may benefit from adaptive lighting, report on our progress and describe our planned future work and evaluation.
\end{abstract}

\section{Author Keywords}

Visual impairment; children; adaptive light; beacons.

\section{ACM Classification Keywords}

K.4.2: Social Issues - Assistive technologies

This is the author's copy of this paper. This paper was presented as a poster at the $9^{\text {th }}$ Nordic Conference on Human-Computer Interaction (NordiCHI '16). Please see the URL below for the definitive version of this paper.

\section{Introduction}

Everyday tasks, like finding clothes and toys, can be a challenge for visually impaired (VI) children, especially young children beginning to explore and learn about the unfamiliar world around them. These tasks are challenging because visual impairment makes it difficult to perceive objects against noisy backgrounds. Lighting areas of rooms is hard, especially when there is little also affects everyday tasks; e.g., finding things in dark 
natural light [8]. Perception of shape and form is often poor, especially from a distance; however, many VI children can perceive light [5], colour and motion [1].

We explore the idea of automatically adapting lighting in the home, using brightness, colour and animation to help VI children with their everyday activities. Adapted lighting can also stimulate vision, which encourages children to use their sight. This is important because it can improve vision [3, 13] and encourages them to explore surroundings [7]. Many assistive technologies for visually impaired people deliberately avoid placing demands on sight, but doing so can be beneficial.

Our ideas build on existing strategies for adapting the home environment to help VI children, although we propose doing this automatically based on the child's behaviour and location. Connected light sources could adjust automatically: to identify items or obstacles, to encourage the child to move, and for engaging visual experiences. This paper presents the development of an adaptive lighting system and gives several scenarios describing how it may help VI children at home.

\section{Background}

Sight plays an important role in early development, providing children with information about the world, the items and people within it, and interactions between them. Developmental importance means VI children should be encouraged to use remaining vision as much as possible $[3,7,13]$. As well as supporting overall development, early use of sight can also improve visual function. Several early intervention programs for VI children found their sight improved significantly more than their untreated peers did $[3,13]$.
At home, VI children can be encouraged to use sight by making it easier for them to see during play and other activities. Hyvärinen [8] recommended choosing toys that glisten and recommended shining lights towards them to increase noticeability and appeal; she also suggested dimming the lighting to make toys easier to see. RNIB and Sense, charities supporting VI people in the UK, make similar recommendations for play $[6,11$, 12]. They suggest giving VI children toys that have a strong contrast or which emit light, as these can be easier to see. Sense further recommend using colourful lighting as this can be exciting [12].

Cornelissen et al. [2] found that VI people identified everyday items more effectively when lighting levels increased, beyond the brightness needed by sighted people. Individual differences and preferences meant that "individually adapted illumination is important" for VI people, so that lighting levels suit the person.

As well as helping VI people see, lighting could also provide other benefits, especially for young children who should be encouraged to explore their surroundings. LaGrow et al. [9] suggested that lighting could be adapted to make rooms more visually interesting for children, increasing their motivation to use their sight. Hyvärinen [7] suggested using active visual stimulation to encourage them to explore toys and spaces, helping to develop their use of vision for gathering information.

Cohen-Maitre et al. [1] investigated visual response to colour and motion in VI children, finding both were perceived well and effectively attracted attention. Motion was particularly attractive and the children were able to identify colours more effectively than shapes. Based on these findings, the authors suggest using colour and 


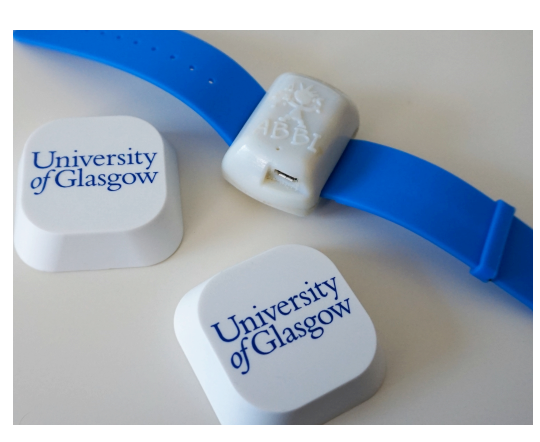

Figure 1: ABBI bracelet prototype and two of the kontakt.io beacons used by our system. Future iterations of the bracelet will have a smaller and more appealing design for children. motion to make it easier for VI children to identify items. For example, by learning associations between colours and items, helping them to find them without identifying their shape in a busy visual scene.

Home environments have been adapted in similar ways by changing the décor and furniture to assist VI children. For example, Lam et al. [10] describe how a VI child's parents redecorated their home so that all walls and floors had a neutral colour, except the child's room, which had blue walls and carpet. These changes helped the child because he could associate the colour blue with his own space and because the light décor throughout the house made it easier for him to see. The work described in this paper builds on these ideas and the previous recommendations by investigating how lighting could be adapted automatically, based on movement and behaviour at home.

Automatically Adapting Lighting in the Home Adapting the lighting based on a child's behaviour means activity and movement sensing is necessary. Our work takes place within the ABBI (Audio Bracelet for Blind Interaction) project [4], which is developing a smart bracelet for VI children. Figure 1 shows an early 3D-printed prototype of the bracelet. It supports Bluetooth communication, has motion sensors, and can produce sound. Its intended use is for rehabilitation activities using movement and sound; however, its processing capabilities mean we could also use it for other things. For example, its sensors and computation capabilities will support the functionality described here. However, the concept of adapted home lighting is not unique to this project and could be implemented using other technologies (like cameras or commodity activity trackers, for example).
Our system will use the ABBI bracelet to detect activity and scan for nearby beacons. Precise localisation within the home is not necessary, as it is sufficient to know which beacons the child is near. As they move, the bracelet will detect nearby beacons and change the lighting based on pre-defined rules. For example, when the system detects that the child leaves their bedroom, it may turn the bedroom lights off. In the following section we present two scenarios which illustrate how this system may work, with examples of how the lighting changes based on activity and movement. Later in the paper, we discuss possible light sources which provide the necessary functionality and we describe our findings based on initial implementation with these lights.

\section{Adaptive Lighting Scenarios}

Scenario 1: Helping to See and Attracting Attention

Jane, a young child with difficulty seeing without bright lighting, walks into her bedroom. As she enters the room, its overhead lights turn on at full brightness, helping her see where the furniture is. A coloured lamp turns on in the corner of the room, illuminating the space where Jane's toys are with a pulsing green light (like Figure 2). She walks towards her toys and all of the lights in the room dim as she sits down. This makes it easier for her to see her toys as they light up against the dark background of her room. As Jane stands up to leave again, the lighting system notices her movement and increases the brightness of the lights again, helping her find a clear path towards the door.

Scenario 2: Enhancing Play with Visualisation

Tom's mother has placed an ABBI bracelet around a new toy she wants him to play with, a ball that makes sounds when it moves. She uses her smartphone to connect the device to a smart lamp in Tom's playroom, 


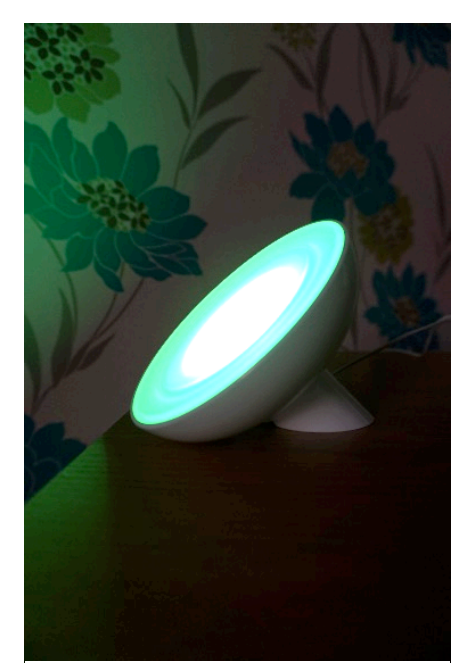

Figure 2: A Philips Hue ${ }^{1}$ table lamp emitting green light.

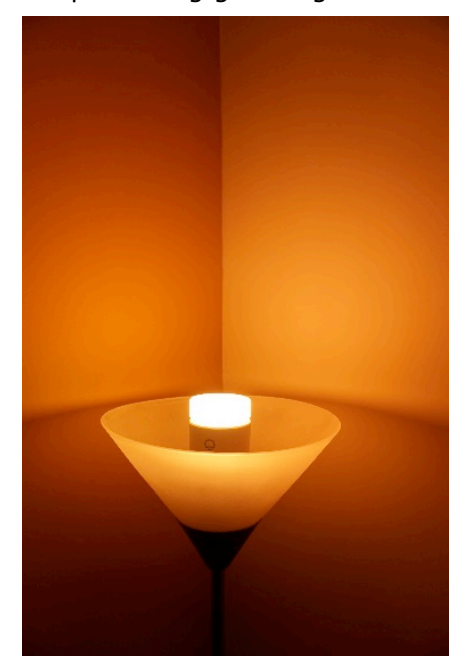

Figure 3: A LIFX ${ }^{2}$ light bulb in a standing lamp. so that the lighting will change in response to the ball moving. When Tom approaches his toys, the ABBI on the ball starts to make sounds to attract his attention. Tom reaches for the ball and as he starts to handle it, the lamp begins to glow in different colours, increasing in brightness as he makes larger movements.

These scenarios show various benefits of automatically adapting the lighting in the home. Lights in Jane's room gave sufficiently bright light (in style of [2]) when necessary, helping her identify obstacles in the room. For people with photophobia (extreme light sensitivity), the opposite could be helpful: i.e., the lights could be dimmed. Coloured light also highlighted Jane's toys, helping create an association between task and colour (like $[1,10])$. Jane's lights dimmed, or switched off, making it easier for her to see visually stimulating toys or objects (as recommended $[6,8,11,12]$ ). Changing the lighting automatically means children can be more independent, as they do not need an adult to reach light switches, and allows the lighting to support various needs (e.g. brighter for movement, darker for play time). Finally, lights produced exciting visual stimuli for Tom as he played with his new toy (as in $[7,9]$ ), which could encourage him to use his vision more actively and to explore new toys visually and with more interest.

The ABBI bracelet was used for different purposes in each scenario: in Scenario 1, Jane's bracelet detected when she entered her bedroom and when she approached her toys; in Scenario 2, Tom's bracelet sensed him approaching the second bracelet his mother had placed on a new toy, and the second bracelet adds motion-sensing and interactive capabilities to the toy, allowing the lamp to respond as Tom played with it.

\section{Current Progress}

We have implemented prototypes that provide the functionality described in our scenarios. At this stage in the ABBI project, the bracelet is unable to scan for beacons, so we have prototyped this functionality using a smartphone. The smartphone scans for beacons in the room and estimates proximity to areas of interest, like the doorway in the first scenario and the toys in the room accordingly. We placed kontakt.io beacons (see Figure 1) at points of interest (like doorways, toys) and estimated proximity using the beacon signal strength. ABBI devices also act as beacons, supporting functionality in Scenario 2. Figure 4 shows an example of how beacons may be arranged in a room; this configuration is not intended to provide precise localisation, but to determine when the ABBI bracelet is near points of interest, like the door or far corner of the room. We used LIFX ${ }^{2}$ bulbs in standard light fittings (like in Figure 3 ) for the lighting. For Scenario 2, we used motion sensor data from the second bracelet to detect when the toy moved and change the light from a lamp in response.

We investigated three types of light source for these prototypes: Philips Hue ${ }^{1}$, LIFX $^{2}$ and an Arduino-based prototype using LED strips ${ }^{3}$. The Arduino system gives the greatest control over the light source as updates happen immediately and every LED is individually controllable. As it contains multiple light sources, it also allows rich animations (for example, light moving back and forth along the light strip). Philips Hue and LIFX products are off-the-shelf, readily available alternatives

\footnotetext{
${ }^{1}$ www.meethue.com Last accessed $12^{\text {th }}$ Sep 2016

${ }^{2}$ www.lifx.com Last accessed $12^{\text {th }}$ Sep 2016

${ }^{3}$ www.adafruit.com/products/1506 Last accessed $12^{\text {th }}$ Sep 2016
} 


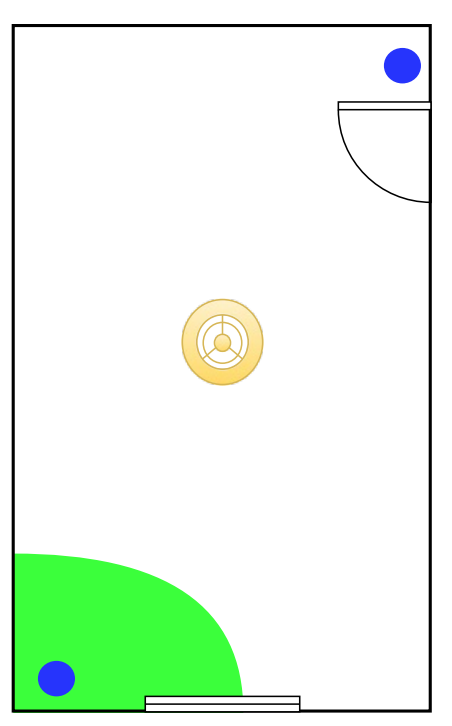

Figure 4: Example beacon placements. Blue circles are beacons, the green arc is a lamp by an item of interest, and the yellow circle in the centre is the overhead light fitting. that can replace or work with existing light fittings and lamps (e.g. Figure 2 and Figure 3). Both have APIs that give control over the hue and brightness of the light. These light sources do not support rich animations based on motion, but could be animated by changing hue and brightness over time. Multiple lights could be used to create illusions of motion; for example, two lamps placed near each other could create the illusion of light moving from side to side, between the lamps.

In developing our prototypes, we found the LIFX light bulbs to be easy to deploy and expressive, as their API provides support for dynamic light changes; e.g. fading between colours and cycling through colours. Lights could use these "animations" to attract attention or create visually exciting stimuli (like in Scenario 1). We attempted to implement similar functionality manually, using both Philips Hue and LIFX lights, but inconsistent response times meant it was difficult to smoothly transition between animation states. For the remainder of this project, we will use the LIFX light bulbs as their animations and compatibility with normal household light fittings meet our requirements.

\section{Future Work: Validation and Evaluation}

Our future work has two aims: to validate and refine our system prototypes, and to identify effective and attractive light designs for VI children. We have had formative discussions with our project partners with expertise in working with VI children, who have given constructive feedback on our ideas and prototypes. Rehabilitators often use torches and light-up toys to stimulate vision, so being able to adapt the lighting automatically in response to these activities could increase their effectiveness. Encouraging use of vision outside of the rehabilitation sessions is also positive, as this contributes to overall visual development.

We have also discussed this system with the wider visual impairment community at events in Scotland. We recently received feedback from attendees at the RNIB Tech Share conference and from members of the Scottish Association for Visual Impairment Education (SAVIE). Discussion with these groups has identified other possible uses for an adaptive lighting system. These include lowering the lighting levels for people with light hypersensitivity, helping VI people find things (e.g. illuminate an area of the room where keys have been left), and reducing family conflicts about lighting and power use by turning unneeded lights off or by dimming them when the room is unoccupied. The latter highlights the importance of being acceptable to other family members: adapting the lighting will also affect them, so lighting systems must consider them as well.

We will continue to validate our system, including more structured discussions with experts and the families of visually impaired children. As home lighting affects everyone in the family, discussion with the latter group are especially important. We are also preparing exploratory studies involving VI children, to investigate how we can best use the design space afforded by commodity lighting products (like the light bulbs used in our prototypes). Although we expect some individual differences (e.g. amount of brightness needed to make obstacles easier to see), we aim to identify colours and light animations which are especially attractive to VI children. Research in this area suggests that colour and motion are attractive visual properties [1]; however, work is needed to identify particularly attractive colours and animations, especially since others have challenged 
previous assumptions about which colours are most preferred by children with a visual impairment [1].

\section{Conclusions}

Everyday tasks and movements can be challenging for VI children, which risks leading to their dependence on others for assistance and which can discourage them from using their remaining sight. In this paper, we described our work towards an adaptive lighting system, which assists VI children by adjusting the light as they move and perform activities around the home. We presented two scenarios describing system functionality and we discussed outcomes from formative discussions with those in the local visually impaired community.

\section{Acknowledgements}

This work is funded by the EU ABBI project (FP7-ICT2013-10 \#611452). We thank those from the VI community in Scotland for their input and feedback.

\section{References}

[1] Stacey Ann Cohen-Maitre and Paul Haerich. 2005. Visual Attention to Movement and Color in Children with Cortical Visual Impairment. Journal of Visual Impairment \& Blindness 99, 7: 1-20.

[2] Frans W. Cornelissen, Annetje Bootsma, and Aart C. Kooijman. 1995. Object Perception by Visually Impaired People at Different Light Levels. Vision Research 35, 1: 161-168.

[3] Elisa Fazzi, Sabrina Giovanna Signorini, S. M. Bova P. Ondei, and P. E. Bianchi. 2005. Early intervention in visually impaired children. International Congress Series 1282: 117-121.

[4] ABBI Project. Last accessed $13^{\text {th }}$ Sep 2016: http://www.abbiproject.eu/

[5] N. M. Flanagan, A. J. Jackson, and A. E. Hill. 2003. Visual impairment in childhood: Insights from a com- munity-based survey. Child: Care, Health and Development 29, 6: 493-499.

[6] Briony Higgins. 2013. Sensory development resource boxes. Last accessed $6^{\text {th }}$ Jan 2016 :

https://www.rnib.org.uk/sites/default/files/sensory_dev elopment_resource_boxes[1].doc

[7] Lea Hyvärinen. 2011. Visually Impaired Children with Multiple Disabilities. Last accessed $6^{\text {th }}$ Jan 2016: http://drleahyvarinen.com/2011/01/14/visuallyimpaired-children-with-multiple-disabilities/

[8] Lea Hyvärinen. 2011. Development of Vision Impaired Children without Other Impairments. Last accessed $6^{\text {th }}$ Jan 2016:

http://drleahyvarinen.com/2011/01/12/developmentof-vision-impaired-children-without-other-impairments/

[9] Steven J. LaGrow, Jin-Pang Leung, S. Leung, and P. Leung. 1998. The Effects on Visually Impaired Children of Viewing Fluorescent Stimuli Under Black-light Conditions. Journal of Visual Impairment \& Blindness 92, 5 : 313-321.

[10] Fook Chang Lam, Fiona Lovett, and Gordon N. Dutton. 2010. Cerebral Visual Impairment in Children: A Longitudinal Case Study of Functional Outcomes Beyond the Visual Acuities. Journal of Visual Impairment \& Blindness 104, 10: 625-635.

[11] RNIB. Toys \& Play for children who are blind or partially sighted. Last accessed $6^{\text {th }}$ Jan 2016:

https://www.rnib.org.uk/sites/default/files/family_frien ds_carers_toys_and_play.pdf

[12] Sense. 2012. Sensory environments for deafblind children and adults: www.sense.org.uk

[13] Patricia M. Sonksen, Aviva Petrie, and Kristina J. Drew. 1991. Promotion of visual development of severely visually impaired babies: evaluation of a developmentally based programme. Developmental Medicine and Child Neurology 33, 4: 320-335. 